dukte für den elektronischen Absatz, der Auswahl der geeigneten Zielgruppe sowie der möglichen Strategieoptionen für den OnlineAuftritt auseinander. Im Rahmen der Preispolitik werden im darauffolgenden Abschnitt die Preissetzung, die Preisfindung sowie die Möglichkeiten zum Preisvergleich vorgestellt. Neben der Darstellung der Vertriebsziele im elektronischen Absatz widmet sich Kapitel 4 insbesondere dem Vertriebsprozess und den Fragen des Vertriebsmanagements. Abschließend werden die kommunikationspolitischen Möglichkeiten zur Kundengewinnung, -bewertung und -bindung präsentiert. Eine besondere Stellung nehmen hierbei die spezifischen Instrumente des Online-Marketing ein, die ausführlich dargestellt werden.

Nach einer umfassenden Einführung in die Grundlagen des elektronischen Absatzes werden die damit verbundenen Herausforderungen für die einzelnen Bereiche des Marketing-Mix in besonders anwendungsbezogener und fokussierter Weise erläutert. Kollmann schafft es mit seinem Buch - ganz im Sinne der Buchreihe „Kohlhammer Edition Marketing“ - eine sehr kompakte und problemorientierte Übersicht über die zentralen Fragestellungen und Problemfelder des Online-Marketing zu geben. Das Buch stellt somit nicht nur für Studierende der BWL, Wirtschaftsinformatik und Medienwissenschaften eine wertvolle Lektüre dar, sondern auch für Praktiker, die ihr Wissen rund um den Themenkomplex Online-Marketing erweitern bzw. vertiefen möchten.

V. Walter

\section{Strategisches Marketingmanagement}

Schaper, Thorsten: Strategisches Marketingmanagement - Einführung in Theorie und Praxis, Göttingen: ForschungsForum, 2008, 188 Seiten, EUR (D) 22,00, ISBN 978-3-937411-24-8

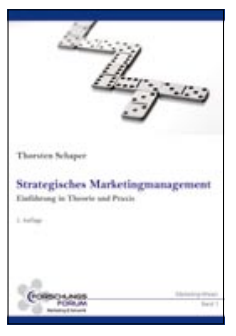

Unternehmen bewegen sich in einer sehr komplexen und dynamischen Umwelt, die zu vielfältigen Marktentwicklungen führt. Entscheidungsträger können sich auf diese Entwicklungen mit einer systematisch-strategischen Ausrichtung des Marketing erfolgreich einstellen. Das strategische Marketingmanagement hat trotz der
Fülle an Veröffentlichungen über gegenwärtige Marketingtrends (Stichworte: Markenführung, Neuromarketing) nichts an Aktualität und Bedeutung verloren.

Mittlerweile in der zweiten Auflage ist das Lehrbuch von Thorsten Schaper erschienen, das als Einführung in die Theorie und Praxis des strategischen Marketingmanagements einen zusammenfassenden Überblick über das strategische Standardwissen gibt. Einem kurzen einführenden Grundlagenteil folgt ein ausführliches Kapitel über die Umwelt- und Unternehmensanalyse, die den Ausgangspunkt strategischer Entscheidungen bildet. Anhand theoretischer Konzepte und diverser Praxisbeispiele erfährt der Leser hier alles Wissenswerte über Analyse, Prognose und Segmentierung von Märkten sowie über die Analyse von Branchen, Wettbewerbern, Abnehmern (insbesondere der Kaufentscheidung von Unternehmen) und des eigenen Unternehmens (Stärken-Schwächen/Chancen-Risiken). Das darauf folgende Kapitel befasst sich mit den Marketingzielen. Es geht auf die Zielbeziehungen ein und gibt wichtige Hinweise zur konkreten Zielformulierung, an der sich der Strategieerfolg messen muss. Das umfangreiche vierte Kapitel diskutiert systematisch die verschiedenen strategischen Handlungsoptionen. Zunächst werden die vier Marktfeldstrategien Marktdurchdringung, Marktentwicklung, Produktentwicklung und Diversifikation dargestellt, gefolgt von verschiedenen Methoden zur Marktfeldwahl. Daran schließen sich weitere strategische Dimensionen in Form der Wettbewerbs- und Positionierungsstrategien sowie der Marktbearbeitungs- und Marktarealstrategien an. Den Abschluss bildet ein kurzes Kapitel zum Marketingcontrolling.

Insgesamt kann ein einführendes Lehrbuch sicherlich keine vertiefenden Einblicke in die Vielfalt des strategischen Marketing gewähren. Hilfreich sind deshalb die Literaturempfehlungen, die sich jeweils am Ende eines jeden Kapitels befinden. Für Praktiker ist dieses Buch insbesondere dann empfehlenswert, wenn man sich einen gut strukturierten Überblick über die strategischen Aufgaben und Handlungsoptionen verschaffen möchte. Primär richtet sich das Buch jedoch an Studierende an Hochschulen und Berufsakademien, für die es nicht zuletzt auch wegen der vielen Beispiele und Lernfragen am jeweiligen Kapitelende empfehlenswert ist.

P. Hehn

\section{Medien- und Internetmanagement}

Wirtz, Bernd W:: Medien- und Internetmanagament, 6. Aufl., Wiesbaden: Gabler, 2008, 831 Seiten, CHF 85,00 / EUR (D) 49,90, ISBN 978-3-8349-0864-3

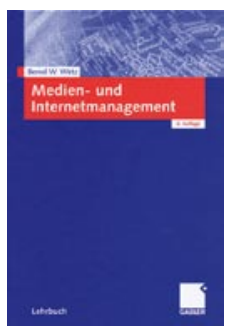

Informations- und Kommunikationstechnologien haben die Welt über die letztenJahrehinwegrevolutioniert. Kaufen, Verkaufen, Produzieren und Informieren - kaum ein Bereich, der nicht verändert worden wäre. Da sich auch die Technologien selbst und deren Nutzung dynamisch und rasant verändern, hat auch Bernd Wirtz sein umfassendes Grundlagenwerk zum Medienund Internetmanagement nun bereits in der sechsten Auflage veröffentlicht. Wirtz bleibt in seinem Werk nicht bei einer Analyse der Entwicklungen stehen, sondern liefert vielmehr eine gut strukturierte Aufarbeitung der Herausforderungen der neuen Medienwelt.

In drei Schritten beschäftigt sich Wirtz mit den Grundlagen des Medienmanagements, den Eigenschaften acht einzelner Medienmärkte und den Herausforderungen des internationalen, integrierten Medienkonzerns. Im ersten Teil erhält der Leser einen Überblick über die technologiegetriebenen Entwicklungen und deren Auswirkungen auf die Märkte und Akteure. Bewährte Konzepte helfen dabei, ein strukturiertes und fundiertes Verständnis zu entwickeln. Diese Konzepte werden im zweiten Teil wieder aufgegriffen, um acht verschiedene Medienmärkte von Zeitungen über Fernsehen bis hin zum Internet aus Managementsicht zu analysieren. Wirtz behandelt die Medienformen nach einem festen Schema; er beschreibt den jeweiligen Markt, die Leistungsspektren, die Besonderheiten der Managementfelder und liefert abschließend ein anschauliches Beispiel. Der dritte Teil des Buches behandelt das zunehmend bedeutsame Medienmanagement über Länderund Medienproduktgrenzen hinweg.

„Medien- und Internetmanagement“ ist ein Grundlagenwerk, das sowohl Übersicht als auch den angemessenen Tiefgang bietet. Das Buch richtet sich an Studierende und Wissenschaftler, die sich einen Zugang zu den Märkten erschließen wollen, wie auch an Praktiker, die nach einer Strukturierung ihres Umfeldes suchen.

L. Ebersbach 